\title{
SPORT RESULTS DYNAMICS IN AGE ASPECT OF BULGARIAN 110M HURDLERS
}

\author{
Grigor Gutev
}

National Sports Academy "Vassil Levski"

Summary

The 110-m hurdle running is a key discipline in each athletic competition due to the facts that it is the first hurdle discipline in historic plan and the spectacular height of the hurdles $-106.7 \mathrm{~cm}$. We assume that analyzing the best Bulgarian hurdlers of all time we can help improvement of selection and training processes control. The aim of the following study is to analyze the sport development of the best Bulgarian $110 \mathrm{~m}$ hurdlers. For the purposes of the study we gathered information regarding their best results during their sport careers - all record holders and hurdlers left trace in the discipline are included in the study. All data was processed using Microsoft Excel and SPSS 19.0 applying variance and correlation analysis. The study results present the specific model characteristics of the sport result valid for Bulgarian hurdlers, such as sport realization and talent development, etc. Based on research data we developed evaluation tables for sport result realization based on respondents - ready for immediate use in sport practice. Also as a help for practice we present regression models for sport results prognosis. Also, we present information which must be used as a role model for sport development of future world elite hurdlers.

Key words: 110 m hurdles, men, age, sport results, dynamics,

\section{Introduction}

The 110-m hurdle running is a key discipline in each athletic competition due to the facts that it is the first hurdle discipline in historic plan and the spectacular height of the hurdles $-106,7 \mathrm{~cm}$.

Due to various anthropometric, climate, geographical, and sport-methodical differences in the different geographical regions we assume that there will be differences in sport realization in the discipline 110 -m hurdlers for men. Based on our studies regarding the sport realization of world elite hurdlers we aimed our attention to the Bulgarian elite in the hurdle running to find its characteristics (Guteva, Gutev, Dimova, 2017).

We assume that analyzing the performance in age aspect of best Bulgarian hurdlers of all time we can help improvement of selection and training processes control and from there - hurdlers sport realization.

\section{Aim and Objectives of the study}

The aim of the following study is to analyze the sport development in age aspect of the best Bulgarian $110 \mathrm{~m}$ hurdlers. For the purposes of the study we gathered information regarding their best results during their sport careers - all record holders and hurdlers left trace in the discipline are included in the study (including athletes with result better than $14,30 \mathrm{sec}$ - in total 37 respondents). In this study, we analyzed the sport result development of all Bulgarian national record holders and the athletes with visible trace in the discipline. All respondents finished their sport careers.

\section{Methods}

The information regarding the personal best results of all respondents is based on developments in this field of Vangelov $(2007,2011)$, „Leka Atletika” sport magazine from the period 2008-2016 and the official web page of Bulgarian Athletic Federation (BAF).

All data was processed using Microsoft Excel and SPSS 19.0 applying variance and correlation analysis. For developing evaluation tables, we used sigmal and T-scale statistical methods.

\section{Results and Discussion}

The study results present the specific model characteristics of the sport result valid for Bulgarian hurdlers, such as sport realization and talent development, etc. 
Table 1

Research data variance analysis.

\begin{tabular}{|c|c|c|c|c|c|c|c|c|c|c|c|c|c|c|c|c|c|c|}
\hline & \multirow{2}{*}{ age PB } & \multicolumn{17}{|c|}{ age } \\
\hline & & 16 & 17 & 18 & 19 & 20 & 21 & 22 & 23 & 24 & 25 & 26 & 27 & 28 & 29 & 30 & 31 & 32 \\
\hline count & 37 & 3 & 9 & 21 & 27 & 28 & 31 & 31 & 29 & 20 & 17 & 17 & 14 & 12 & 9 & 6 & 4 & 1 \\
\hline $\min$ & 19 & 15,58 & 14,19 & 13,99 & 13,93 & 13,79 & 13,54 & 13,59 & 13,33 & 13,43 & 13,41 & 13,46 & 13,67 & 13,59 & 13,83 & 13,77 & 14,57 & \\
\hline $\max$ & 29 & 15,99 & 15,73 & 15,45 & 15,53 & 15,20 & 14,97 & 15,05 & 15,35 & 14,64 & 14,98 & 14,77 & 14,86 & 14,88 & 15,37 & 15,87 & 16,08 & \\
\hline average & 23,11 & 15,81 & 14,80 & 14,74 & 14,64 & 14,47 & 14,34 & 14,32 & 14,33 & 14,27 & 14,22 & 14,25 & 14,25 & 14,41 & 14,60 & 14,62 & 15,12 & 14,42 \\
\hline$m \bar{x}$ & 0,371 & 0,12 & 0,16 & 0,08 & 0,08 & 0,07 & 0,07 & 0,06 & 0,07 & 0,07 & 0,10 & \begin{tabular}{|l|}
0,09 \\
\end{tabular} & \begin{tabular}{|l|}
0,10 \\
\end{tabular} & 0,11 & 0,18 & 0,30 & 0,33 & \\
\hline$S$ & 2,26 & 0,21 & 0,47 & 0,38 & 0,43 & 0,39 & 0,37 & 0,36 & 0,40 & 0,31 & 0,39 & 0,38 & 0,39 & 0,39 & 0,54 & 0,74 & 0,67 & \\
\hline V\% & 9,77 & 1,33 & 3,18 & 2,58 & 2,96 & 2,72 & 2,55 & 2,51 & 2,81 & 2,14 & 2,77 & 2,64 & 2,72 & 2,67 & 3,73 & 5,07 & 4,41 & \\
\hline As & 0,42 & & 1,27 & 0,16 & 0,13 & 0,35 & $-0,22$ & \begin{tabular}{|l|}
$-0,22$ \\
\end{tabular} & $-0,16$ & \begin{tabular}{|l|}
$-1,36$ \\
\end{tabular} & $-0,13$ & $-0,41$ & $-0,01$ & $-0,81$ & 0,02 & 0,83 & 1,59 & \\
\hline Ex & 0,11 & & 1,65 & $-0,36$ & $-0,78$ & $-0,50$ & $-0,64$ & $-0,51$ & 1,28 & 1,73 & $-0,03$ & $-0,38$ & $-1,15$ & 0,10 & $-1,57$ & 1,04 & 2,74 & \\
\hline
\end{tabular}

On Table 1 we present the variance analysis for all respondents. We find best, weakest and average result for each age - from 16 to 32 years of age. Most cases we find for the period 18-28 years (range from 31 to 12 athletes per age). The best average values and from there the best period for setting personal best and revealing personal sport abilities in the hurdle running in Bulgaria is between 21 and 27 years. We must note that this is not mandatory and the sport development of each athlete has individual character. But the tendencies will be a good guide for the people working with youngsters. Based on the skewness (As) and kurtosis (Ex) indexes we found normal data distribution in the period 17-27, 29 and 31.

On Figure 1 we see graphic of the best, weakest and average result of best Bulgarian hurdlers in the age period 16-31 years. From this data it is clearly visible the dynamics of sport result thought different age periods.

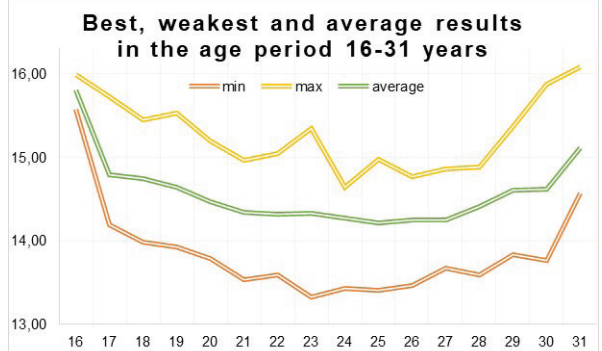

Figure 1.

On Table 2 and Figure 2 we see the correlation between the sport result for the certain age and athletes personal best result. Based on this we found the age result for 22, 24-28 have higher interrelations with the $\mathrm{PB}$, and from there this age period is best for setting high level of sport results in the discipline $110 \mathrm{~m}$ hurdles.
Table 2

Correlation matrix - interrelations between PB and sport result for the certain age.

\begin{tabular}{|c|c|c|c|c|}
\hline \multirow{2}{*}{ 兽 } & \multirow{2}{*}{ PB } & \multirow{2}{*}{$\begin{array}{l}\text { number } \\
\text { of cases }\end{array}$} & \multicolumn{2}{|c|}{ Correlation is } \\
\hline & & & 0,05 level & 0,01 level \\
\hline 18 & 0,440 & 21 & 0,41 & 0,53 \\
\hline 19 & 0,530 & 27 & 0,37 & 0,48 \\
\hline 20 & 0,604 & 28 & 0,36 & 0,46 \\
\hline 21 & 0,695 & 31 & 0,35 & 0,45 \\
\hline 22 & 0,841 & 31 & 0,35 & 0,45 \\
\hline 23 & 0,687 & 29 & 0,36 & 0,46 \\
\hline 24 & 0,708 & 20 & 0,42 & 0,54 \\
\hline 25 & 0,787 & 17 & 0,46 & 0,58 \\
\hline 26 & 0,758 & 17 & 0,46 & 0,58 \\
\hline 27 & 0,878 & 14 & 0,5 & 0,62 \\
\hline 28 & 0,804 & 12 & 0,53 & 0,66 \\
\hline
\end{tabular}

Correlation between PB and age (period 18-28 years)

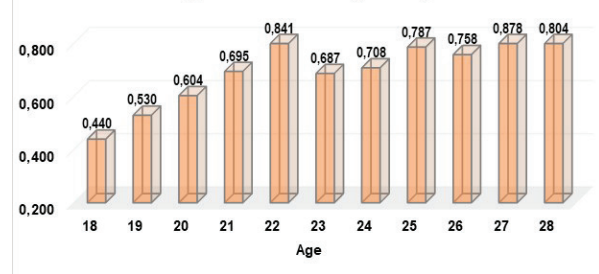

Figure 2.

Based on research data we developed evaluation tables for sport result realization based on respondents - ready for immediate use in sport practice. In the development of assessment tables, we included only age with normal data distribution based on the variance analysis.

On Table 3 we present the first way of assessment which can be directly implemented in sport practice. Using 7 level evaluation scale we can assess the evolution of sport result in age aspect based on the results of all Bulgarian hurdlers with $\mathrm{PB}$ result under $14,50 \mathrm{sec}$. 
Table 3

Assessment using the Sigmal method based on 7 level evaluation scale.

\begin{tabular}{|c|c|c|c|c|c|c|c|}
\hline assesment & 17 & 18 & 19 & 20 & 21 & 22 & 23 \\
\hline very high & under 13,86 & under 13,98 & under 13,78 & under 13,69 & under 13,60 & under 13,60 & under 13,53 \\
\hline high & $13,86-14,32$ & $13,98-14,35$ & $13,78-14,20$ & $13,69-14,07$ & $13,60-13,96$ & $13,60-13,95$ & $13,53-13,92$ \\
\hline above average & $14,33-14,56$ & $14,36-14,54$ & $14,21-14,42$ & $14,08-14,27$ & $13,97-14,15$ & $13,96-14,13$ & $13,93-14,12$ \\
\hline average & $14,57-15,04$ & $14,55-14,93$ & $14,43-14,86$ & $14,28-14,67$ & $14,16-14,53$ & $14,14-14,50$ & $14,13-14,53$ \\
\hline below average & $15,05-15,27$ & $14,94-15,12$ & $14,87-15,07$ & $14,68-14,86$ & $14,54-14,71$ & $14,51-14,68$ & $14,54-14,73$ \\
\hline low & $15,28-15,74$ & $15,13-15,50$ & $15,08-15,50$ & $14,87-15,25$ & $14,72-15,08$ & $14,69-15,04$ & $14,74-15,13$ \\
\hline \multirow[t]{2}{*}{ very low } & over 15,74 & over 15,50 & over 15,50 & over 15,25 & over 15,08 & over 15,04 & over 15,13 \\
\hline & 24 & 25 & 26 & 27 & 29 & 31 & \\
\hline very high & under 13,65 & under 13,44 & under 13,49 & under 13,47 & under 13,52 & under 13,78 & \\
\hline high & $13,65-13,95$ & $13,44-13,82$ & $13,49-13,86$ & $13,47-13,85$ & $13,52-14,05$ & $13,78-14,44$ & \\
\hline above average & $13,96-14,11$ & $13,83-14,02$ & $13,87-14,05$ & $13,86-14,05$ & $14,06-14,32$ & $14,45-14,78$ & \\
\hline average & $14,12-14,43$ & $14,03-14,42$ & $14,06-14,44$ & $14,06-14,45$ & $14,33-14,87$ & $14,79-15,46$ & \\
\hline below average & $14,44-14,58$ & $14,43-14,61$ & $14,45-14,63$ & $14,46-14,64$ & $14,88-15,14$ & $15,47-15,79$ & \\
\hline low & $14,59-14,89$ & $14,62-15,00$ & $14,64-15,01$ & $14,65-15,03$ & $15,15-15,68$ & $15,80-16,46$ & \\
\hline very low & over 14,89 & over 15,00 & over 15,01 & over 15,03 & over 15,68 & over 16,46 & \\
\hline
\end{tabular}

We offer another way for assessment of sport For example, in the age of 19 years a result close to development in age aspect using T-scale and Z-scores. $\quad 14,64$ is average ( $\mathrm{Z}$ score is 0,0 and $\mathrm{T}$ score is 50 ).

Table 4

Assessment using T-scale and Z-scoring.

\begin{tabular}{|c|c|c|c|c|c|c|c|c|c|c|c|c|c|c|c|}
\hline $\mathbf{P \%}$ & $\mathbf{Z}$ & $\mathbf{T}$ & $\mathbf{1 7}$ & $\mathbf{1 8}$ & $\mathbf{1 9}$ & $\mathbf{2 0}$ & $\mathbf{2 1}$ & $\mathbf{2 2}$ & $\mathbf{2 3}$ & $\mathbf{2 4}$ & $\mathbf{2 5}$ & $\mathbf{2 6}$ & $\mathbf{2 7}$ & $\mathbf{2 9}$ & $\mathbf{3 1}$ \\
\hline $\mathbf{9 9 , 8 7}$ & $\mathbf{3 , 0}$ & $\mathbf{8 0}$ & 13,39 & 13,60 & 13,34 & 13,28 & 13,24 & 13,24 & 13,12 & 13,35 & 13,04 & 13,12 & 13,08 & 12,97 & 13,11 \\
\hline $\mathbf{9 9 , 3 8}$ & $\mathbf{2 , 5}$ & $\mathbf{7 5}$ & 13,62 & 13,79 & 13,56 & 13,48 & 13,43 & 13,42 & 13,32 & 13,51 & 13,23 & 13,31 & 13,28 & 13,24 & 13,45 \\
\hline $\mathbf{9 7 , 7 2}$ & $\mathbf{2 , 0}$ & $\mathbf{7 0}$ & 13,86 & 13,98 & 13,77 & 13,68 & 13,61 & 13,60 & 13,52 & 13,66 & 13,43 & 13,50 & 13,47 & 13,51 & 13,78 \\
\hline $\mathbf{9 3 , 3 2}$ & $\mathbf{1 , 5}$ & $\mathbf{6 5}$ & 14,09 & 14,17 & 13,99 & 13,88 & 13,79 & 13,78 & 13,72 & 13,81 & 13,63 & 13,69 & 13,67 & 13,79 & 14,11 \\
\hline $\mathbf{8 4 , 1 3}$ & $\mathbf{1 , 0}$ & $\mathbf{6 0}$ & 14,33 & 14,36 & 14,21 & 14,07 & 13,98 & 13,96 & 13,93 & 13,97 & 13,83 & 13,88 & 13,86 & 14,06 & 14,45 \\
\hline $\mathbf{6 9 , 1 5}$ & $\mathbf{0 , 5}$ & $\mathbf{5 5}$ & 14,56 & 14,55 & 14,42 & 14,27 & 14,16 & 14,14 & 14,13 & 14,12 & 14,02 & 14,06 & 14,06 & 14,33 & 14,78 \\
\hline $\mathbf{5 0 , 0 0}$ & $\mathbf{0 , 0}$ & $\mathbf{5 0}$ & 14,80 & 14,74 & 14,64 & 14,47 & 14,34 & 14,32 & 14,33 & 14,27 & 14,22 & 14,25 & 14,25 & 14,60 & 15,12 \\
\hline $\mathbf{3 0 , 8 5}$ & $\mathbf{- 0 , 5}$ & $\mathbf{4 5}$ & 15,03 & 14,93 & 14,85 & 14,66 & 14,53 & 14,50 & 14,53 & 14,42 & 14,42 & 14,44 & 14,44 & 14,88 & 15,45 \\
\hline $\mathbf{1 5 , 8 7}$ & $\mathbf{- 1 , 0}$ & $\mathbf{4 0}$ & 15,27 & 15,12 & 15,07 & 14,86 & 14,71 & 14,68 & 14,73 & 14,58 & 14,61 & 14,63 & 14,64 & 15,15 & 15,78 \\
\hline $\mathbf{6 , 6 8}$ & $\mathbf{- 1 , 5}$ & $\mathbf{3 5}$ & 15,50 & 15,31 & 15,29 & 15,06 & 14,89 & 14,86 & 14,93 & 14,73 & 14,81 & 14,82 & 14,83 & 15,42 & 16,12 \\
\hline $\mathbf{2 , 2 8}$ & $\mathbf{- 2 , 0}$ & $\mathbf{3 0}$ & 15,74 & 15,50 & 15,50 & 15,26 & 15,08 & 15,04 & 15,13 & 14,88 & 15,01 & 15,00 & 15,03 & 15,69 & 16,45 \\
\hline $\mathbf{0 , 6 2}$ & $\mathbf{- 2 , 5}$ & $\mathbf{2 5}$ & 15,97 & 15,70 & 15,72 & 15,45 & 15,26 & 15,22 & 15,33 & 15,04 & 15,20 & 15,19 & 15,22 & 15,96 & 16,78 \\
\hline $\mathbf{0 , 1 3}$ & $\mathbf{- 3 , 0}$ & $\mathbf{2 0}$ & 16,21 & 15,89 & 15,94 & 15,65 & 15,44 & 15,40 & 15,54 & 15,19 & 15,40 & 15,38 & 15,41 & 16,24 & 17,12 \\
\hline
\end{tabular}

\section{Conclusion}

Based on our study based, on the experience of best Bulgarian hurdlers (with result under 14,50 sec.) we analyzed the sport realization model and specific periods for reaching maximum of individual sport abilities.

Based on research data we developed two ways of sport result assessment and from there sport specialists can make better plans and corrections of the training process and sport realization.

However, often sport realization is characterized by athlete individuality, so this must be taken in mind when applying the results of the following study. 


\section{References}

Guteva, Hr., G. Gutev, I. Dimova, Sport results dynamics in age aspect in the discipline $110 \mathrm{~m}$ hurdles - men, Proceedings of XI International Scientific and Practical Conference of Students and Young Scientists "Modern University Sport Science", May 17-18, 2017, Moscow, 2017. (page 90-96).

http://bfla.org/ - Official web site of the Bulgarian Athletic Federation.

Vangelov, A. BFLA, Leka Atletika - Darjavni shampioni, Medalisti I Bulgarski ranglisti 1926-2006g. Es Print, Sofia, 2007g. // Вангелов, А., БФЛА, Лека атлетика - Държавни шампиони, Медалисти и Български ранглисти 1926-2006 г., Ес Принт, София, 2007 г.
Vangelov, A., Nai, nai, nai v Bulgarskata atletika, TipTop Pres, Sofia, 2011g. // Вангелов, А., Най, най, най в Българската атлетика, ТипТоп Прес, София, 2011 г. Journal "Leka Atletika", 2008-2016g. // Сп. Лека атлетика, 2008-2016 г.

\section{Author information:}

Senior lecturer Grigor Gutev, PhD, National Sports Academy "Vassil Levski”, Department "Track \& Field” Address: Sofia, Bulgaria, ZIP 1700, "Studentski gard", NSA "Vassil Levski”, office 334 (Department “Track \& Field")

Mobile +359 889503 604, e-mail: grigor.gutev@gmail. com 